\title{
Proteomic systems evaluation of the molecular validity of preclinical psychosis models compared to schizophrenia brain pathology
}

David A. Cox ${ }^{1}$, Michael G. Gottschalk ${ }^{1}$, Hendrik Wesseling ${ }^{1}$, Agnes Ernst ${ }^{1}$, Jason D. Cooper ${ }^{1}$, Sabine Bahn ${ }^{1}$

${ }^{1}$ Department of Chemical Engineering and Biotechnology, University of Cambridge, Cambridge, CB2 1QT, United Kingdom.

\section{Corresponding Author:}

Sabine Bahn, MD, PhD.

Department of Chemical Engineering and Biotechnology, University of Cambridge, Cambridge, CB2 1QT, United Kingdom

Email: sb209@cam.ac.uk

\section{Abstract}

Pharmacological and genetic rodent models of schizophrenia play an important role in the drug discovery pipeline, but quantifying the molecular similarity of such models with the underlying human pathophysiology has proved difficult. We developed a novel systems biology methodology for the direct comparison of anterior prefrontal cortex tissue from four established glutamatergic rodent models and schizophrenia patients, enabling the evaluation of which model displays the greatest similarity to schizophrenia across different pathophysiological characteristics of the disease.

Liquid chromatography coupled tandem mass spectrometry $\left(L C-M S^{E}\right)$ proteomic profiling was applied comparing healthy and "disease state" in human post-mortem samples and rodent brain tissue samples derived from models based on acute and chronic phencyclidine (PCP) treatment, ketamine treatment or NMDA receptor knockdown. Protein-protein interaction networks were constructed from significant abundance changes and enrichment analyses enabled the identification of five functional domains of the disease such as "development and differentiation", which were represented across all four rodent models and were thus subsequently used for cross-species comparison. Kernel-based machine learning techniques quantified that the chronic PCP model represented schizophrenia brain changes most closely for four of these functional domains.

This is the first study aiming to quantify which rodent model recapitulates the neuropathological features of schizophrenia most closely, providing an indication of face validity as well as potential guidance in the refinement of construct and predictive validity. The methodology and findings presented here support recent efforts to overcome translational hurdles of preclinical psychiatric research by associating functional dimensions of behaviour with distinct biological processes.

\section{Keywords}

schizophrenia; systems biology; psychosis models; molecular validity; chronic phencyclidine (PCP) rat model; proteomics 


\section{Introduction}

Schizophrenia (SCZ) affects approximately $1 \%$ of the world's population and has been listed as one of the top ten causes of disease-related disabilities in the world (Rössler et al., 2005; "WHO | The global burden of disease: 2004 update,"). Antipsychotics represent the first line of pharmacotherapy for SCZ and predominantly target dopamine, noradrenaline and serotonin pathways (Tandon et al., 2008). However these medications typically fail to treat the cognitive and negative symptoms, which contribute substantially to the morbidity of SCZ (Buchanan and Gold, 1996; Green, 1996; Greenwood et al., 2005). Due to increased evidence that dysfunction of glutamatergic transmission is implicated in psychotic states (Konradi and Heckers, 2003; Olney and Farber, 1995; Tsai and Coyle, 2002), glutamatergic animal models of the disease have increasingly been used to test the potential efficacy of novel compounds (Bondi et al., 2012).

Glutamatergic mechanisms were initially implicated in SCZ pathogenesis after reduced cerebrospinal fluid levels were reported in patients (Kim et al., 1980), with later studies pointing to more complex mechanisms behind dysfunction of glutamate neurotransmission (Javitt and Zukin, 1991). Glutamatergic models of SCZ in animals include genetic manipulation of the N-methyl-D-aspartate (NMDA) receptor (NMDAR) (Halene et al., 2009) and acute or chronic exposure to NMDAR antagonists such as phencyclidine (PCP) (Jentsch and Roth, 1999) and ketamine (Kapur and Seeman, 2002). Systemic treatment with these antagonists in preclinical studies was found to mimic negative symptoms of the disease alongside an increase in glutamate efflux in the prefrontal cortex (Liu and Moghaddam, 1995; Moghaddam et al., 1997). Administration of PCP and ketamine in clinical studies was found to induce psychotomimetic effects ranging from positive symptoms such as hallucinations and paranoia (Javitt and Zukin, 1991) to negative and cognitive symptoms in healthy volunteers (Grayson et al., 2014; Neill et al., 2014), in addition to precipitating psychotic relapses in chronic stable SCZ patients (Lahti et al., 1995).

Preclinical evaluation is an important step in the drug discovery pipeline and allows the prioritization of compounds for clinical trials. Regardless of ongoing advances in other areas of medicine, the development of preclinical models for neuropsychiatric disorders is at a near standstill (Nestler and Hyman, 2010a). Additionally, there have been failures in finding new pharmacological treatments because the observed outcomes in behavioural screenings were not predictive of clinical outcomes. The main reason for this is the fact that uniquely human behavioural symptoms cannot be reliably or convincingly mirrored or assessed in animals, explaining difficulties in face validity. On the other hand pharmacological validity actually selects for models based on the mechanisms of actions of already known compounds. Finally, the face validity of genetic and pharmacological models has been criticized as more than a single gene is considered to be important in the etiology of SCZ and injections of compounds only induce transient phenotypes. Notwithstanding these challenges, there are now attempts to identify molecular disease hallmarks of distinct endophenotypes in neuropsychiatric animal models, as an essential stage of their integration into the drug development pipeline (Nestler and Hyman, 2010a).

Rodents are the most common animal model used,normally coming from a breeding background with low heterogeneity and have the potential for highly controlled experimental settings with the added benefit of providing a source of freshly collected cerebral tissue samples (Turck et al., 2005). Although it is difficult or even impossible to mirror the entire symptom spectrum of a psychiatric 
disorder in a given animal model, insights into molecular endophenotypes have yielded important information into the underlying pathophysiology (Kendler and Neale, 2010). Such models enable the study of central biological processes associated with a particular group of symptoms and the testing and evaluation of novel treatments. In this study four established rodent models of SCZ based on either psychosis-inducing NMDAR-antagonists (Ketamine and PCP) or genetic modifications targeting the glutamate system (NR1-knockdown) were investigated. Rats were chosen over mice in the pharmacological models to increase brain tissue yield for proteomic extractions. Mice were chosen for the NR1 knockdown due to the availability of superior methods of genetic manipulation compared to rats. Here, we have integrated the proteomic information of human SCZ post mortem brains and four rodent models of the disease in a novel systems biology approach. The anterior frontal cortex was chosen as the tissue of interest in both humans and rodents as a brain region which is strongly linked to psychotic disorders. The anterior prefrontal cortex plays a crucial role in the processing and evaluation of internally generated information across multiple cognitive operations (Ramnani and Owen, 2004). The negative and cognitive symptoms of schizophrenia are characterized by impairments in executive functioning and socio-emotional cognition.

The biological processes affected in the anterior frontal cortices in each condition were characterized and compared across species and models. Based on protein-protein-interaction networks we identified key functional patterns that allowed us to quantify the molecular similarity of the models with the human condition, introducing a novel way to interrogate translational preclinical validity.

\section{Materials and Methods}

Figure 1 provides a step by step illustration of the methodology behind this analysis.

\subsection{Clinical Samples}

46 post-mortem anterior prefrontal cortex (BA10) brain samples were obtained from the Stanley Medical Research Institute (Torrey et al., 2000). Samples were derived from 23 SCZ patients and 23 control (CT) subjects.

Tissue collection took place with full informed consent of a first-degree relative to comply with the Declaration of Helsinki. As outlined previously (Gottschalk et al., 2014), there were no differences in grey and white matter volumes between samples, and no significant differences in brain side, gender or secondary axis diagnosis of alcohol abuse/dependency and drug abuse/dependency between patients and controls.

Four glutamatergic animal models of SCZ commonly used in preclinical drug discovery and development were generated applying the protocols outlined below. Rats (Rattus norvegicus) were used for the ketamine, acute PCP (aPCP) and chronic PCP (cPCP) models and mice (Mus musculus) were used for the NR1 knockdown model. All animals were housed 4-5 per cage on a 12-h light/dark cycle in a temperature-controlled facility with food and water available ad libitum. At the end of each protocol, rodents were sacrificed ( $n=X$ to $Y$, per group) by decapitation, and brain tissue samples were collected. A matching number of control samples were collected the same time.

Ketamine: $0.9 \%$ sterile saline $(n=10)$ and $10 \mathrm{mg} / \mathrm{kg}$ ketamine $(n=10)$ injections were administered subcutaneously as described previously (Wesseling et al., 2015b). Dosage levels were based on 
previous research which examined dose/response levels based on locomotor activity ataxia, brain dialysis/neurotransmitter release and pharmacological magnetic resonance data (Gastambide et al., 2013; Li et al., 2014; Littlewood et al., 2006; Smith et al., 2011). Doses were chosen which yielded robust readouts while avoiding inducing anaesthesia. Sacrifice and tissue collection took place two hours after the last injection.

aPCP: A subcutaneous dose of saline $(n=10)$ or $5 \mathrm{mg} / \mathrm{kg}$ PCP hydrochloride $(n=10)$ was administered as previously described (Ernst et al., 2012; Palmowski et al., 2014). Sacrifice and tissue collection took place $30 \mathrm{~min}$ after the last injection.

cPCP: Subcutaneous doses of saline $(n=10)$ or $5 \mathrm{mg} / \mathrm{kg}$ PCP hydrochloride $(\mathrm{n}=9)$ were administered for 15 consecutive days as previously described (Wesseling et al., 2015c). Sacrifice and tissue collection took place 30min after the last injection on day 15. NR1 Knockdown: NR1 transgenic mice were bred and genotyped as previously described (Halene et al., 2009; Mohn et al., 1999; Wesseling et al., 2014). 12 adult male homozygous mice and 12 wild-type littermates were used for this study.

\subsection{Label-Free LC-MS ${ }^{\mathrm{E}}$ Analysis of Brain Tissue}

Using previously defined storage, preparation and measurement procedures (Gottschalk et al., 2014), brain samples were analyzed individually in technical duplicates using label-free LC-MS ${ }^{\mathrm{E}}$. For the subsequent analysis, a human proteome database was obtained from UniProt (retrieved 201514-10, number of entries 20,196). A joint Mus musculus and Rattus norvegicus database was created by merging the protein sequences of the respective UniProt reference proteome files (retrieved 2015-14-10, number of entries 24,664). MS raw data and ProteinLynx Global Server v2.5 search results were imported into the Rosetta Elucidator software (build 3.3, Rosetta Biosoftware). Settings and procedures were applied as stated previously (Gottschalk et al., 2014), and peptide signal intensities for each sample were exported for pre-processing and statistical analysis in $R(R$ Development Core Team, 2013). Only peptides with an amino acid sequence ending in $\mathrm{R}$ or K were considered to avoid unspecific trypsin cleavage. Non-unique peptides were excluded. Sample outliers were identified using the first two principal components (Beniger et al., 1980) resulting in the removal of one control sample from the aPCP, CPCP, Ketamine and NR1-knockdown models.

Following $\log _{2}$ transformation to stabilize data variance, protein abundance changes for the human and rodent model comparisons were determined using a linear model, with a stepwise selection adjustment for covariates age, gender, diagnoses of alcohol or substance abuse, post-mortem interval (PMI), brain $\mathrm{pH}$ and brain side. We analyzed the data using a fixed effects linear model and allowed for the duplicate measurements using a robust variance estimator (Freedman, 2006).

For the SCZ tissue, the false discovery rate (FDR) was controlled at a cut-off of 0.05 by adjusting the $p$-values according to the Benjamini Hochberg procedure (Benjamini and Hochberg, 1995). Due to the small sample sizes for each rodent model, a null distribution was empirically generated to obtain the actual distribution of $p$-values (Ernst, 2004). This was done by comparing the ranking of a test statistic from the original model to those obtained through 10,000 random permutations of the sample status.

\subsection{Protein-Protein Interaction (PPI) Networks}


An analysis framework was designed to compare SCZ and rodent tissue on a functional level, based on the annotation of Gene Ontology (GO) terms to the protein abundance changes described earlier. In order for these terms to represent the biophysical interactions which occur between sets of proteins (Stelzl et al., 2005), PPI networks for SCZ and the APCP, CPCP, ketamine and NR1 knockdown rodent models were created using the software package Cytoscape v3.2.1 (Cline et al., 2007). Each network was represented as a graph where the nodes are proteins and the edges are interactions between proteins.

Networks were constructed by retrieving all available known PPI information for proteins with significantly changed abundances following multiple testing or permutations as described in 2.2 , and their first-order protein interactors, from the databases MINT (Zanzoni et al., 2002), IntAct (Hermjakob et al., 2004) and UniProt (Apweiler et al., 2004). Filtering was applied to both the node and edge lists for all four networks. Nodes were filtered by taxonomy identifiers ( 9606 for Homo sapiens in the SCZ network, in addition to 10116 and 10090 for Rattus norvegicus and Mus musculus respectively in the rodent networks), while edges were filtered to exclude all connections other than direct interactions or physical associations between proteins. All unconnected subsets of nodes were removed from the network. The structures of each network were assessed using four common properties of complex networks, clustering coefficient, average degree, characteristic path length and density.

\subsubsection{GO term enrichment}

GO term enrichment was computed on each PPI network using the ClueGO (Bindea et al., 2009) Cytoscape package, with default settings unless described below. The ontology category used was "Biological Process". The Homo sapiens GO database was used for the terms of the SCZ network. The Mus musculus database was used to evaluate all rodent networks to reduce a species-specific annotation bias. A two-sided hypergeometric distribution was used to compute the statistical significance of each GO term, describing the probabilities associated with sampling randomly without replacement from a finite network of proteins where all proteins have an equal chance of being drawn. This determines whether any GO terms occur at a frequency greater than would be expected by chance. For each term, $p$-values were corrected for multiple testing ( $q$-values) by applying a Benjamini-Hochberg correction. Terms with a significant $q$ value $(q<0.05)$ were taken forward and terms with no significant enrichment $(q>0.05)$ or less than two proteins were removed automatically.

\subsubsection{Kappa Score Grouping}

The list of terms for each network were functionally grouped based on shared underlying proteins using a kappa score (Huang et al., 2007) of 0.7. The kappa score metric reflects the degree of the relationship between two $\mathrm{GO}$ terms. A score of 0.7 or higher requires abundant shared proteins (Bindea et al., 2009; Merico et al., 2010), ensuring that the groups are likely to be biologically similar. Functional groups which did not contain at least two GO terms were excluded from the analysis. Groups were named according to the most significant (q-value $<=0.05$ ) GO term.

\subsubsection{Local Linear Embedding Kernel Group Augmentation}


The biological interpretation of each functional group was enhanced by augmenting the groups with closely related GO terms using a diffusion-type manifold embedding technique called a Local Linear Embedding (LLE) kernel, commonly used to group related proteins or genes (Li et al., 2010; Zare et al., 2011). Kernels are manifold embedding techniques which are commonly used in bioinformatics to classify data points into particular categories (Lerman and Shakhnovich, 2007). In this instance, kernel methods are used to compute similarity metrics through a geometric interpretation of manifold embedding where each GO term is treated as though positioned in a virtual twodimensional space based on its place in the GO tree. The LLE kernel was chosen over other kernel techniques, as it emphasizes short-range interactions between terms (Roweis and Saul, 2000). For every term in a given functional group, a positive similarity value between that term and any other term in the $\mathrm{GO}$ database meant this new term was related and could be added to the group. $\mathrm{A}$ negative value meant that the new term was unrelated.

For each SCZ term, the LLE kernel was used to compute the pairwise similarity metric to every other term in the Homo sapiens GO tree. Likewise with each aPCP, CPCP, ketamine and NR1-knockdown term, similarity metrics were computed to every term in the Mus musculus GO tree. The concept behind the use of the LLE kernel to augment the existing functional groups is that the particular proteins underlying these additional terms will be associated with a variety of related biological functions to those already conveyed by the group. Hence, this method yields more informative functional groups of highly related terms.

\subsection{Functional Comparison between SCZ and Rodent Models}

The enhanced groups of GO terms were used as the basis for a functional comparison between SCZ and the four rodent models. The percentage overlap of terms in terms of $Z$ score was computed between each of the SCZ groups and the rodent model groups. Hierarchical clustering using Ward's criterion was employed to identify related clusters of groups for both $\mathrm{SCZ}$ and each model, enabling the identification of different functional domains in the disease represented across all four models, where each domain is a vector of $\mathrm{GO}$ terms. The subsets of $\mathrm{GO}$ terms behind each domain which are completely unique to that domain were then identified, thus defining the biological functionality more precisely.

\subsection{Domain Comparison Through GO Term Similarity}

Having identified a series of unique functional domains of human SCZ represented by the four models, it was necessary to quantify which model represented each domain most closely. This was done by modifying an approach from genetic research (Fröhlich et al., 2006; Speer et al., 2005) to obtain a numerical quantification for the closeness of the models to the disease by computing a similarity score between rodent and human domains. These scores were obtained through evaluating the average of the best matching $\mathrm{GO}$ term similarity between the domain vectors, where the pairwise similarity scores between GO terms were obtained using the LLE kernel described in 2.3.3. The closer the similarity scores between rodent and human domains are to 1 , the more similar they are, while the closer the scores are to 0 , the more dissimilar they are.

\section{Results}

\subsection{Behavioural Characteristics of Rodent Models}


For both acute and chronic models, PCP doses were found to induce the expected abnormalities of hyperlocomotion, increased stereotypic behaviour and impaired attention and social interaction as reported previously (Lee et al., 2005; Sams-Dodd, 1996). Ketamine doses induced hyperlocomotion, stereotypy, impaired information processing with abnormalities in cognitive function, and impaired social interaction, behavioural characterizations which has been described in previous acute ketamine models (Becker et al., 2003; Gastambide et al., 2013). NR1-knockdown mice displayed both hyperlocomotion and increased stereotypic behaviour in addition to impairments in cognition and escape behaviours as found previously (Dzirasa et al., 2009). 3.2. Protein Abundance Changes

\section{for Brain Tissue Comparisons}

A total of 1280 quantifiable proteins were measured across all SCZ brain samples, 643 across all aPCP samples, 873 across all cPCP samples, 772 across all Ketamine samples and 409 across all NR1 knockdown samples. Supplementary Figure 1 summarizes the overlap in proteins measured between these groups. Based on linear modelling, we found that 159 proteins were differentially expressed in SCZ patients compared to CT individuals, following false discovery correction. Linear modelling of the rodent models found 47 proteins to be differently expressed in the aPCP-control comparison, 84 in the CPCP-control comparison, 93 in the Ketamine-control comparison and 80 in the NR1 knockdown-control comparison, following permutation testing. (Supplementary Tables S1$\mathrm{S} 5$ display these proteins and their fold changes).

\subsection{PPI Networks and GO Term Enrichment Analysis}

PPI networks were created for SCZ and all four rodent models by PPI information from the UniProt, MINT and IntAct protein databases between the significant proteins identified in 3.2, and their respective first-order interactors. The significantly changed abundances of these proteins was determined through multiple hypotheses testing (human samples) or permutation testing (rodent samples). All rodent model PPI networks, as well as the SCZ PPI network were characterised using several structural properties of complex networks - clustering coefficient, average degree, characteristic path length and density (Table 1).

Table 1. Structural properties of PPI Networks

\begin{tabular}{|c|c|c|c|c|c|c|c|c|}
\hline $\begin{array}{l}\text { Condition/ } \\
\text { Model }\end{array}$ & N & $n(\%$ of $N)$ & $\begin{array}{l}\text { Number of } \\
\text { nodes }\end{array}$ & $\begin{array}{l}\text { Number of } \\
\text { edges }\end{array}$ & $\begin{array}{l}\text { Clustering } \\
\text { coefficient }\end{array}$ & $\begin{array}{l}\text { Average } \\
\text { degree }\end{array}$ & $\begin{array}{l}\text { Characteristic } \\
\text { path length }\end{array}$ & Density \\
\hline $\mathrm{SCZ}$ & 159 & 115 (72\%) & 2163 & 3297 & 0.009 & 2.698 & 4.162 & 0.001 \\
\hline $\mathrm{aPCP}$ & 48 & $29(62 \%)$ & 150 & 203 & 0.002 & 2.493 & 3.772 & 0.017 \\
\hline $\mathrm{CPCP}$ & 85 & $59(69 \%)$ & 351 & 507 & 0.017 & 2.741 & 3.3 & 0.008 \\
\hline Ketamine & 94 & $54(57 \%)$ & 189 & 271 & 0.039 & 2.698 & 4.278 & 0.014 \\
\hline $\begin{array}{l}\text { NR1knockd } \\
\text { own }\end{array}$ & 81 & $52(64 \%)$ & 349 & 537 & 0.019 & 2.951 & 3.368 & 0.008 \\
\hline
\end{tabular}

The structural properties of average degree and characteristic path length are most closely related between the SCZ and Ketamine networks. The CPCP network displays the greatest tendency to form clusters to the disease network, indicating that this model has the greatest functional similarities to the disease. Functional enrichment analysis of the networks, and grouping of the resulting terms 
according to a Kappa score of 0.7 , yielded 222 SCZ functional groups, 63 aPCP groups, 128 CPCP groups, 117 Ketamine groups and 119 NR1-knockdown groups, with each group corresponding to a specific biological process. Table 2 displays the top 5 functional groups for SCZ and each rodent model, in order of significance.

Table 2. Top 5 functional groups for each PPI Network

\begin{tabular}{|c|c|c|c|c|c|}
\hline $\begin{array}{l}\text { Condition/ } \\
\text { Model }\end{array}$ & N & Top five group names & $\begin{array}{l}\text { No of GO } \\
\text { terms }\end{array}$ & Group p-value & $\begin{array}{l}\text { No of significant } \\
\text { proteins per group }\end{array}$ \\
\hline \multirow[t]{5}{*}{ SCZ } & \multirow[t]{5}{*}{2163} & Regulation of phosphorus metabolic process & 14 & 4.7 E-115 & 24 \\
\hline & & Phosphorylation & 12 & $6.4 \mathrm{E}-92$ & 23 \\
\hline & & Protein phosphorylation & 4 & $1.1 \mathrm{E}-70$ & 38 \\
\hline & & Cellular protein metabolic process & 4 & $6.2 \mathrm{E}-68$ & 40 \\
\hline & & Intracellular signal transduction & 3 & $2.1 \mathrm{E}-67$ & 30 \\
\hline \multirow[t]{5}{*}{ aPCP } & \multirow[t]{5}{*}{150} & Cellular component assembly & 3 & $1.2 \mathrm{E}-16$ & 6 \\
\hline & & Transport & 3 & 4.0 E-15 & 14 \\
\hline & & Regulation of cellular metabolic process & 23 & $4.2 \mathrm{E}-15$ & 14 \\
\hline & & Negative regulation of biological process & 2 & 5.3 E-14 & 7 \\
\hline & & Regulation of signalling & 4 & $6.4 \mathrm{E}-14$ & 4 \\
\hline \multirow[t]{5}{*}{$\mathrm{CPCP}$} & \multirow[t]{5}{*}{351} & Phosphorus metabolic process & 2 & $1.6 \mathrm{E}-40$ & 31 \\
\hline & & Organic substance catabolic process & 3 & $2.4 \mathrm{E}-40$ & 30 \\
\hline & & Single-organism catabolic process & 23 & $3.2 \mathrm{E}-40$ & 31 \\
\hline & & Transport & 2 & $2.2 \mathrm{E}-32$ & 33 \\
\hline & & Cell projection organization & 15 & $1.7 \mathrm{E}-25$ & 21 \\
\hline \multirow[t]{5}{*}{ Ketamine } & \multirow[t]{5}{*}{189} & Transport & 2 & $1.4 \mathrm{E}-26$ & 20 \\
\hline & & Regulation of localization & 2 & $2.3 \mathrm{E}-20$ & 10 \\
\hline & & Cell communication & 2 & $2.7 \mathrm{E}-19$ & 22 \\
\hline & & Cell-cell signalling & 2 & $5.1 \mathrm{E}-19$ & 6 \\
\hline & & Regulation of cell communication & 4 & $5.2 \mathrm{E}-19$ & 13 \\
\hline \multirow{5}{*}{$\begin{array}{l}\text { NR1knockd } \\
\text { own }\end{array}$} & \multirow[t]{5}{*}{349} & Phosphorus metabolic process & 2 & $3.5 \mathrm{E}-34$ & 28 \\
\hline & & Transport & 2 & $1.3 \mathrm{E}-31$ & 21 \\
\hline & & Establishment of localization in cell & 9 & $3.4 \mathrm{E}-23$ & 14 \\
\hline & & Cellular component assembly & 3 & $1.7 \mathrm{E}-22$ & 15 \\
\hline & & Regulation of transport & 2 & $6.0 \mathrm{E}-22$ & 13 \\
\hline
\end{tabular}

\subsection{Identification of Corresponding Functional Domains Between SCZ and Rodent Models}

Following the enhancement of these functional groups by kernel techniques, the percentage overlap was computed between SCZ and rodent model groups. It was found that groups which clustered together and overlapped are involved in closely related biological processes, resulting in the identification of five functional domains of the disease - "development and differentiation", "intracellular signalling and regulation", "intracellular transport and organization", "biosynthetic processes and energy metabolism", and "nucleic acid metabolism and ATP/GTPase activity" - which are represented across all four models. These domains are shown in Figure 2. 
PPI network edges for the significant proteins underlying these domains, as identified in 3.2 , are listed in Supplementary Tables S6-S10.

\subsection{Identification of Most Representative Rodent Model via Similarity Based Methods}

Similarity-based methods were used to compare the vectors of $\mathrm{GO}$ terms for each domain, making it possible to quantify the model that is most representative of SCZ through similarity scores (Table 3).

Table 3. Domain Comparison to SCZ Based on Similarity Scores Computed From Feature Space Embedding

\begin{tabular}{|c|c|c|c|c|}
\hline Functional Domain & aPCP & CPCP & Ketamine & $\begin{array}{l}\text { NR1 } \\
\text { Knockdown }\end{array}$ \\
\hline Development and differentiation & 0.398 & 0.561 & 0.405 & 0.445 \\
\hline Intracellular signalling and regulation & 0.365 & 0.385 & 0.454 & 0.396 \\
\hline Intracellular transport and organization & 0.639 & 0.688 & 0.644 & 0.496 \\
\hline Biosynthetic processes and energy metabolism & 0.582 & 0.61 & 0.573 & 0.554 \\
\hline Nucleic acid metabolism and ATP/GTPase activity & 0.68 & 0.681 & 0.648 & 0.658 \\
\hline
\end{tabular}

These scores indicate that overall the CPCP model represents SCZ the most closely for the four functional domains, "development and differentiation", "intracellular transport and organization", "biosynthetic processes and energy metabolism", and "nucleic acid metabolism and ATP/GTPase activity," more than the other three models. The Ketamine model represents "intracellular signalling and regulation" most closely, although the similarity scores between models and the disease were generally not so close for this domain (less than 0.5 ).

4. Discussion Our current understanding of the underlying molecular pathology associated with SCZ is limited. Post-mortem studies and animal models of the disease can provide new insights into the patterns of alterations at the genetic and protein level which play a role in the neuropathology of SCZ (Chan et al., 2014; Gottschalk et al., 2014; Mäki et al., 2005). However, so far it has proven hard to quantify the molecular similarity of the models with the human disease pathology (Nestler and Hyman, 2010b). These characterization issues have meant that progress has been limited in developing new pharmacotherapies from animal studies (Fonio et al., 2012). As a consequence, few new chemical entities have reached the clinic over the last decades.

In the present study we employed a non-hypothesis driven integration of proteomic data on the systems biology level, a methodology which enables the direct comparison of brain changes from four rodent models with those observed in human disease brains. This makes it possible to evaluate which of the given models represents SCZ most closely for different pathophysiological aspects of the disorder. Previous approaches have tried to establish various phenotypic similarities between animal models and SCZ through construct, face and predictive validity, assessments predominantly based on behavioural paradigms often supplemented with histological or electrophysiological investigations (Nestler and Hyman, 2010b). However, these approaches have struggled to establish a particular model as the 'best for use' for a particular aspect of the disease because assessing similarities between animal behaviour and patient characteristics is typically vague, leading to difficulties regarding bias and irreproducibility (Tordjman et al., 2007). In addition, demonstrating 
that molecular changes in SCZ blood serum or plasma alone are equivalent to that of a relevant animal model has proven to be challenging (Kluge et al., 2011).

Hence, rather than relying on a comparison based solely on the significantly altered proteins as measured through $\mathrm{LCMS}^{\mathrm{E}}$, this method seeks to compare both human and animal tissue at a functional level using GO terms annotated to proteins. Conducting a proteomic analysis through the construction of protein-protein interaction networks has advantages over a genomic/transcriptomic based approach, as this provides a greater indication of the functional alterations within tissue (Bayés and Grant, 2009). Additionally, while the heritability of schizophrenia has been approximated as being between $50 \%$ and $90 \%$ based on twin studies, the genetic landscape may not be the best framework for comparison as SCZ appears to be a polygenic disorder with disease phenotypes arising through the accumulation of multiple small risk genes (Kendler and Diehl, 1993; Maier, 2008; Sullivan et al., 2003). A protein-protein interaction network based approach was chosen over pathway analysis to characterize the biological processes more precisely through the inclusion of first-degree interactors in these networks. Once functional groups had been determined within the network, an approach commonly used to compare gene functionality was adopted (Huang et al., 2012; Plaisier et al., 2012; Poos et al., 2013). This approach utilized kernel methods, one of the most advanced techniques in machine learning, to compare vectors of $\mathrm{GO}$ terms through their closeness to each other in the $\mathrm{GO}$ hyperspace, thus making individual comparisons possible between models and the disease (Lerman and Shakhnovich, 2007; Schlicker et al., 2006).

Collectively, this method enabled the identification of five functional aspects identified in SCZ postmortem brain tissue which are represented across all four animal models -intracellular signalling and regulation, development and differentiation, intracellular transport and localization, biosynthetic processes and energy metabolism, nucleic acid metabolism and ATP/GTPase activity. Our approach revealed that the latter four functional domains are represented most closely by the CPCP model. Blocking the NMDA receptor through PCP treatment has been previously associated with neurodegenerative pathologies in both humans and animal models (Lei et al., 2008), but while acute NMDA antagonist treatment has mainly been linked to disinhibition of the cortical transmitters glutamate, dopamine and serotonin (5-HT) (Adams and Moghaddam, 1998; López-Gil et al., 2007), chronic NMDA antagonist administration is associated with more complex molecular and behavioural adaptations, leading to a more defined cognitive deficit profile (Jentsch, 1997; Olney et al., 1989). This could explain why the CPCP model outperforms the ketamine and aPCP models across most categories. Reduced rates of oxygen uptake into mitochondria isolated from brain tissue have been observed in previous PCP rat studies, hence it is not unexpected that chronic PCP treatment represented impairments in energy metabolism, a common trait of psychiatric diseases (Konradi et al., 2004; Prabakaran et al., 2004; Rajasekaran et al., 2015). The significantly changed protein sets which underlie each of these functional domains in the chronic PCP model could form the basis of future assays for drug development using techniques such as selective reaction monitoring (SRM). Once such candidate biomarkers have been identified, SRM can be applied to analyze protein abundance levels of these markers in tissue in a quantitative manner. We have previously reported on this in a study of anterior prefrontal cortex tissue from patients with SCZ, bipolar disorder and major depressive disorder, in which the expression levels were quantified for a panel of 56 proteins suggested to be associated with various functional aspects of these disorders, including for examplealterations in cellular energy metabolism and dysfunction of neuronal differentiation (Wesseling et al., 2015a). It is interesting to note that despite precautions of introducing a species- 
specific bias (e.g. joint rodent protein database, one shared functional annotation pool) the NR1 knockdown mice showed the lowest similarity scores across the domains. Although all rodent models investigated in this study support the theory of a dysfunctional hypoglutamatergic frontal cortex state in SCZ post mortem brains (Gottschalk et al., 2015) we cannot exclude the possibility that compensatory developmental mechanisms following a single gene knockdown are more likely to influence and potentially dilute a functional cross-species similarity, than the molecular reaction of the frontal cortex to NMDA-receptor antagonist exposure in adult animals.

From a systems biological perspective, this study has several strengths including the use of carefully matched post-mortem brain samples while adjusting for covariates in a regression analysis via a stepwise selection procedure which accounted for the most commonly addressed confounders while avoiding overparameterization. The present approach involved the use of a joint rodent protein database at the $L C M S^{E}$ processing stage and the Mus musculus GO database at the GO term enrichment stage to avoid introducing any species-specific bias which could bias the results in favour of one particular model. The chosen systemic methodology is superior to the standard way of pathway analysis based on individual proteins which is most commonly used in preclinical studies. A systems approach leverages the signature proteins as a representation of changes in signalling pathways, instead of interpreting the relevance between each protein and phenotype. By tackling a pressing question in translational research through the adaptation and application of machine learning based methods already established as a means of conducting functional comparisons in other disciplines of medicine, decision processes in preclinical neuropsychiatry could benefit of synergies between different fields of molecular research. While the study did not account for directionality in terms of protein signalling, and the effects on a particular function/process can differ depending on whether the proteins associated with a particular GO term are up/downregulated, there is as yet no standard annotation to account for this in PPI networks. In addition, one has to keep in mind that ultimately the identified domains were limited by the detected fraction of the proteome and therefore are less likely to contain GO terms based on proteins that are difficult to detect in whole-tissue approaches, for example membrane-integral proteins or proteins with very specific expression time windows (e.g. proteins involved in apoptosis signalling). An identification of domains of "underrepresented" GO terms (inversed enrichment analysis) was beyond the scope of this article.

The current consensus regarding animal models of psychiatric disorders is that no single model can completely recapitulate the full complexity of human conditions (Wong and Josselyn, 2015). As an example, auditory electrophysiological abnormalities recorded in studies involving the NR1 knockdown model are thought to more closely resemble those seen in autism than schizophrenia (Gandal et al., 2012). Conversely, this model is a good proxy for behavioural phenotypes which invoke some of the negative symptoms of schizophrenia such as impairments in spatial cognitive performance (Dzirasa et al., 2009) and reduced social interaction. Hence, it is felt that future models of SCZ should be focused on behavioural endophenotypes and more importantly molecular alterations, as we gain more understanding of the genetic and neurodevelopmental causes (Powell and Miyakawa, 2006; Stewart and Kalueff, 2015). In particular given the failures of current medications in treatment of negative and cognitive symptoms of SCZ, preclinical models for different symptom clusters are likely to play an increasingly important role in new pharmacological approaches (Keefe et al., 2007; Sarnyai et al., 2011; Tomasik et al., 2015, 2014). Our results provide evidence that different models can represent functional aspects of SCZ more closely than others, 
however future research should aim to introduce proteomic information of different putative SCZ animal models, most notably dopaminergic manipulations using direct and indirect dopamine agonists which have previously shown to induce behavioural phenotypes associated with positive and negative symptoms of SCZ such as hyperactivity, persisting prepulse inhibition abnormalities and attention deficit (Jones et al., 2011). The flexible structure of the proposed framework allows the reevaluation of cross-species network comparisons once new information is available. The presented analysis is the first to directly compare multiple animal models to SCZ on a functional level, with the findings supporting the notion that a variety of available models, each reflecting different pathological molecular hallmarks of SCZ, could be important for insights into the molecular and cellular basis of behavioural abnormalities relevant to $\mathrm{SCZ}$, as well as testing their responsiveness to existing and upcoming medication. This need will become increasingly important as we seek to reach a broader understanding of the ramifications of a given genetic, environmental or pharmacological manipulation in the context of psychotic spectrum disorders.

\section{Acknowledgement}

This research was supported by the Stanley Medical Research Institute (SMRI) and the NEWMEDS Innovative Medicines Initiative.

\section{References}

Adams, B., Moghaddam, B., 1998. Corticolimbic dopamine neurotransmission is temporally dissociated from the cognitive and locomotor effects of phencyclidine. J. Neurosci. 18, 554554.

Apweiler, R., Bairoch, A., Wu, C.H., Barker, W.C., Boeckmann, B., Ferro, S., Gasteiger, E., Huang, H., Lopez, R., Magrane, M., Martin, M.J., Natale, D.A., O’Donovan, C., Redaschi, N., Yeh, L.-S.L., 2004. UniProt: the Universal Protein knowledgebase. Nucleic Acids Res. 32, D115-D119. doi:10.1093/nar/gkh131

Bayés, A., Grant, S.G.N., 2009. Neuroproteomics: understanding the molecular organization and complexity of the brain. Nat. Rev. Neurosci. 10, 635-646. doi:10.1038/nrn2701

Becker, A., Peters, B., Schroeder, H., Mann, T., Huether, G., Grecksch, G., 2003. Ketamine-induced changes in rat behaviour: A possible animal model of schizophrenia. Prog. Neuropsychopharmacol. Biol. Psychiatry 27, 687-700. doi:10.1016/S0278-5846(03)00080-0

Beniger, J.R., Barnett, V., Lewis, T., 1980. Outliers in Statistical Data. Contemp. Sociol. 9, 560. doi:10.2307/2066277

Benjamini, Y., Hochberg, Y., 1995. Benjamini Y, Hochberg Y. Controlling the false discovery rate: a practical and powerful approach to multiple testing. J. R. Stat. Soc. B 57, 289-300. doi:10.2307/2346101

Bindea, G., Mlecnik, B., Hackl, H., Charoentong, P., Tosolini, M., Kirilovsky, A., Fridman, W.-H., Pagès, F., Trajanoski, Z., Galon, J., 2009. ClueGO: a Cytoscape plug-in to decipher functionally grouped gene ontology and pathway annotation networks. Bioinformatics 25, 1091-1093. doi:10.1093/bioinformatics/btp101

Bondi, C., Matthews, M., Moghaddam, B., 2012. Glutamatergic animal models of schizophrenia. Curr. Pharm. Des. 18, 1593-604.

Buchanan, R.W., Gold, J.M., 1996. Negative symptoms: diagnosis, treatment and prognosis. Int. Clin. Psychopharmacol. 11 Suppl 2, 3-11.

Chan, M.K., Gottschalk, M.G., Haenisch, F., Tomasik, J., Ruland, T., Rahmoune, H., Guest, P.C., Bahn, S., 2014. Applications of blood-based protein biomarker strategies in the study of psychiatric disorders. Prog. Neurobiol. 122, 45-72. doi:10.1016/j.pneurobio.2014.08.002

Cline, M.S., Smoot, M., Cerami, E., Kuchinsky, A., Landys, N., Workman, C., Christmas, R., Avila- 
Campilo, I., Creech, M., Gross, B., Hanspers, K., Isserlin, R., Kelley, R., Killcoyne, S., Lotia, S., Maere, S., Morris, J., Ono, K., Pavlovic, V., Pico, A.R., Vailaya, A., Wang, P.-L., Adler, A., Conklin, B.R., Hood, L., Kuiper, M., Sander, C., Schmulevich, I., Schwikowski, B., Warner, G.J., Ideker, T., Bader, G.D., 2007. Integration of biological networks and gene expression data using Cytoscape. Nat. Protoc. 2, 2366-2382. doi:10.1038/nprot.2007.324

Dzirasa, K., Ramsey, A.J., Takahashi, D.Y., Stapleton, J., Potes, J.M., Williams, J.K., Gainetdinov, R.R., Sameshima, K., Caron, M.G., Nicolelis, M.A.L., 2009. Hyperdopaminergia and NMDA receptor hypofunction disrupt neural phase signaling. J. Neurosci. 29, 8215-24. doi:10.1523/JNEUROSCI.1773-09.2009

Ernst, A., Ma, D., Garcia-Perez, I., Tsang, T.M., Kluge, W., Schwarz, E., Guest, P.C., Holmes, E., Sarnyai, Z., Bahn, S., 2012. Molecular validation of the acute phencyclidine rat model for schizophrenia: identification of translational changes in energy metabolism and neurotransmission. J. Proteome Res. 11, 3704-14. doi:10.1021/pr300197d

Ernst, M.D., 2004. Permutation Methods: A Basis for Exact Inference. Stat. Sci. 19, 676-685.

Fonio, E., Golani, I., Benjamini, Y., 2012. Measuring behavior of animal models: faults and remedies. Nat. Methods 9, 1167-70. doi:10.1038/nmeth.2252

Freedman, D. a, 2006. On The So-Called "Huber Sandwich Estimator" and "Robust Standard Errors." Am. Stat. 60, 299-302. doi:10.1198/000313006X152207

Fröhlich, H., Speer, N., Zell, A., 2006. Kernel Based Functional Gene Grouping, in: Proc. Int. Joint Conf. Neural Networks. pp. 6886-6891.

Gandal, M.J., Anderson, R.L., Billingslea, E.N., Carlson, G.C., Roberts, T.P.L., Siegel, S.J., 2012. Mice with reduced NMDA receptor expression: more consistent with autism than schizophrenia? Genes. Brain. Behav. 11, 740-50. doi:10.1111/j.1601-183X.2012.00816.x

Gastambide, F., Mitchell, S.N., Robbins, T.W., Tricklebank, M.D., Gilmour, G., 2013. Temporally distinct cognitive effects following acute administration of ketamine and phencyclidine in the rat. Eur. Neuropsychopharmacol. 23, 1414-22. doi:10.1016/j.euroneuro.2013.03.002

Gottschalk, M.G., Wesseling, H., Guest, P.C., Bahn, S., 2015. Proteomic enrichment analysis of psychotic and affective disorders reveals common signatures in presynaptic glutamatergic signaling and energy metabolism. Int. J. Neuropsychopharmacol. 18. doi:10.1093/ijnp/pyu019

Gottschalk, M.G., Wesseling, H., Guest, P.C., Bahn, S., 2014. Proteomic enrichment analysis of psychotic and affective disorders reveals common signatures in presynaptic glutamatergic signaling and energy metabolism. Int. J. Neuropsychopharmacol. 18, pyu019. doi:10.1093/ijnp/pyu019

Grayson, B., Adamson, L., Harte, M., Leger, M., Marsh, S., Piercy, C., Neill, J.C., 2014. The involvement of distraction in memory deficits induced by NMDAR antagonism: relevance to cognitive deficits in schizophrenia. Behav. Brain Res. 266, 188-92. doi:10.1016/j.bbr.2014.03.011

Green, M.F., 1996. What are the functional consequences of neurocognitive deficits in schizophrenia? Am. J. Psychiatry 153, 321-30. doi:10.1176/ajp.153.3.321

Greenwood, K.E., Landau, S., Wykes, T., 2005. Negative symptoms and specific cognitive impairments as combined targets for improved functional outcome within cognitive remediation therapy. Schizophr. Bull. 31, 910-21. doi:10.1093/schbul/sbi035

Halene, T.B., Ehrlichman, R.S., Liang, Y., Christian, E.P., Jonak, G.J., Gur, T.L., Blendy, J.A., Dow, H.C., Brodkin, E.S., Schneider, F., Gur, R.C., Siegel, S.J., 2009. Assessment of NMDA receptor NR1 subunit hypofunction in mice as a model for schizophrenia. Genes. Brain. Behav. 8, 661-75. doi:10.1111/j.1601-183X.2009.00504.x

Hermjakob, H., Montecchi-Palazzi, L., Lewington, C., Mudali, S., Kerrien, S., Orchard, S., Vingron, M., Roechert, B., Roepstorff, P., Valencia, A., Margalit, H., Armstrong, J., Bairoch, A., Cesareni, G., Sherman, D., Apweiler, R., 2004. IntAct: an open source molecular interaction database. Nucleic Acids Res. 32, D452-5. doi:10.1093/nar/gkh052

Huang, D.W., Sherman, B.T., Tan, Q., 2007. The DAVID Gene Functional Classification Tool: a novel 
biological module-centric algorithm to functionally analyze large gene lists. Genome Biol. 8, R183. doi:10.1186/gb-2007-8-9-r183

Huang, Y., Chen, H.-C., Chiang, C.-W., Yeh, C.-T., Chen, S.-J., Chou, C.-K., 2012. Identification of a twolayer regulatory network of proliferation-related microRNAs in hepatoma cells. Nucleic Acids Res. 40, 10478-93. doi:10.1093/nar/gks789

Javitt, D.C., Zukin, S.R., 1991. Recent advances in the phencyclidine model of schizophrenia. Am. J. Psychiatry 148, 1301-8. doi:10.1176/ajp.148.10.1301

Jentsch, J.D., 1997. Enduring Cognitive Deficits and Cortical Dopamine Dysfunction in Monkeys After Long-Term Administration of Phencyclidine. Science (80-. ). 277, 953-955. doi:10.1126/science.277.5328.953

Jentsch, J.D., Roth, R.H., 1999. The neuropsychopharmacology of phencyclidine: from NMDA receptor hypofunction to the dopamine hypothesis of schizophrenia. Neuropsychopharmacology 20, 201-25. doi:10.1016/S0893-133X(98)00060-8

Jones, C.A., Watson, D.J.G., Fone, K.C.F., 2011. Animal models of schizophrenia. Br. J. Pharmacol. 164, 1162-94. doi:10.1111/j.1476-5381.2011.01386.x

Kapur, S., Seeman, P., 2002. NMDA receptor antagonists ketamine and PCP have direct effects on the dopamine $\mathrm{D}(2)$ and serotonin 5-HT(2)receptors-implications for models of schizophrenia. Mol. Psychiatry 7, 837-44. doi:10.1038/sj.mp.4001093

Keefe, R.S.E., Bilder, R.M., Davis, S.M., Harvey, P.D., Palmer, B.W., Gold, J.M., Meltzer, H.Y., Green, M.F., Capuano, G., Stroup, T.S., McEvoy, J.P., Swartz, M.S., Rosenheck, R.A., Perkins, D.O., Davis, C.E., Hsiao, J.K., Lieberman, J.A., 2007. Neurocognitive effects of antipsychotic medications in patients with chronic schizophrenia in the CATIE Trial. Arch. Gen. Psychiatry 64, 633-47. doi:10.1001/archpsyc.64.6.633

Kendler, K.S., Diehl, S.R., 1993. The genetics of schizophrenia: a current, genetic-epidemiologic perspective. Schizophr. Bull. 19, 261-85.

Kendler, K.S., Neale, M.C., 2010. Endophenotype: a conceptual analysis. Mol. Psychiatry 15, 789-97. doi:10.1038/mp.2010.8

Kim, J.S., Kornhuber, H.H., Schmid-Burgk, W., Holzmüller, B., 1980. Low cerebrospinal fluid glutamate in schizophrenic patients and a new hypothesis on schizophrenia. Neurosci. Lett. 20, 379-82.

Kluge, W., Alsaif, M., Guest, P.C., Schwarz, E., Bahn, S., 2011. Translating potential biomarker candidates for schizophrenia and depression to animal models of psychiatric disorders. Expert Rev. Mol. Diagn. 11, 721-33. doi:10.1586/erm.11.61

Konradi, C., Eaton, M., MacDonald, M.L., Walsh, J., Benes, F.M., Heckers, S., 2004. Molecular evidence for mitochondrial dysfunction in bipolar disorder. Arch. Gen. Psychiatry 61, 300-8. doi:10.1001/archpsyc.61.3.300

Konradi, C., Heckers, S., 2003. Molecular aspects of glutamate dysregulation: implications for schizophrenia and its treatment. Pharmacol. Ther. 97, 153-79.

Lahti, A.C., Koffel, B., LaPorte, D., Tamminga, C.A., 1995. Subanesthetic doses of ketamine stimulate psychosis in schizophrenia. Neuropsychopharmacology 13, 9-19. doi:10.1016/0893133X(94)00131-I

Lee, P.R., Brady, D.L., Shapiro, R.A., Dorsa, D.M., Koenig, J.I., 2005. Social interaction deficits caused by chronic phencyclidine administration are reversed by oxytocin. Neuropsychopharmacology 30, 1883-94. doi:10.1038/sj.npp.1300722

Lei, G., Xia, Y., Johnson, K.M., 2008. The role of Akt-GSK-3beta signaling and synaptic strength in phencyclidine-induced neurodegeneration. Neuropsychopharmacology 33, 1343-53. doi:10.1038/sj.npp.1301511

Lerman, G., Shakhnovich, B.E., 2007. Defining functional distance using manifold embeddings of gene ontology annotations. Proc. Natl. Acad. Sci. U. S. A. 104, 11334-11339. doi:10.1073/pnas.0702965104

Li, J., Ishiwari, K., Conway, M.W., Francois, J., Huxter, J., Lowry, J.P., Schwarz, A.J., Tricklebank, M., 
Gilmour, G., 2014. Dissociable effects of antipsychotics on ketamine-induced changes in regional oxygenation and inter-regional coherence of low frequency oxygen fluctuations in the rat. Neuropsychopharmacology 39, 1635-44. doi:10.1038/npp.2014.10

Li, L., Ching, W., Chan, Y., Mamitsuka, H., 2010. On network-based kernel methods for proteinprotein interactions with applications in protein functions prediction. J. Syst. Sci. Complex. 23, 917-930. doi:10.1007/s11424-010-0207-y

Littlewood, C.L., Jones, N., O’Neill, M.J., Mitchell, S.N., Tricklebank, M., Williams, S.C.R., 2006. Mapping the central effects of ketamine in the rat using pharmacological MRI. Psychopharmacology (Berl). 186, 64-81. doi:10.1007/s00213-006-0344-0

Liu, J., Moghaddam, B., 1995. Regulation of glutamate efflux by excitatory amino acid receptors: evidence for tonic inhibitory and phasic excitatory regulation. J. Pharmacol. Exp. Ther. 274, 1209-15.

López-Gil, X., Babot, Z., Amargós-Bosch, M., Suñol, C., Artigas, F., Adell, A., 2007. Clozapine and haloperidol differently suppress the MK-801-increased glutamatergic and serotonergic transmission in the medial prefrontal cortex of the rat. Neuropsychopharmacology 32, 208797. doi:10.1038/sj.npp.1301356

Maier, W., 2008. Common risk genes for affective and schizophrenic psychoses. Eur. Arch. Psychiatry Clin. Neurosci. 258 Suppl , 37-40. doi:10.1007/s00406-008-2008-z

Mäki, P., Veijola, J., Jones, P.B., Murray, G.K., Koponen, H., Tienari, P., Miettunen, J., Tanskanen, P., Wahlberg, K.-E., Koskinen, J., Lauronen, E., Isohanni, M., 2005. Predictors of schizophrenia--a review. Br. Med. Bull. 73-74, 1-15. doi:10.1093/bmb/ldh046

Merico, D., Isserlin, R., Stueker, O., Emili, A., Bader, G.D., 2010. Enrichment map: a network-based method for gene-set enrichment visualization and interpretation. PLoS One 5, e13984. doi:10.1371/journal.pone.0013984

Moghaddam, B., Adams, B., Verma, A., Daly, D., 1997. Activation of glutamatergic neurotransmission by ketamine: a novel step in the pathway from NMDA receptor blockade to dopaminergic and cognitive disruptions associated with the prefrontal cortex. J. Neurosci. 17, 2921-7.

Mohn, A.R., Gainetdinov, R.R., Caron, M.G., Koller, B.H., 1999. Mice with reduced NMDA receptor expression display behaviors related to schizophrenia. Cell 98, 427-36.

Neill, J.C., Harte, M.K., Haddad, P.M., Lydall, E.S., Dwyer, D.M., 2014. Acute and chronic effects of NMDA receptor antagonists in rodents, relevance to negative symptoms of schizophrenia: a translational link to humans. Eur. Neuropsychopharmacol. 24, 822-35. doi:10.1016/j.euroneuro.2013.09.011

Nestler, E.J., Hyman, S.E., 2010a. Animal models of neuropsychiatric disorders. Nat. Neurosci. 13, 1161-9. doi:10.1038/nn.2647

Nestler, E.J., Hyman, S.E., 2010b. Animal models of neuropsychiatric disorders. Nat. Neurosci. 13, 1161-9. doi:10.1038/nn.2647

Olney, J.W., Farber, N.B., 1995. Glutamate receptor dysfunction and schizophrenia. Arch. Gen. Psychiatry 52, 998-1007.

Olney, J.W., Labruyere, J., Price, M.T., 1989. Pathological changes induced in cerebrocortical neurons by phencyclidine and related drugs. Science $244,1360-2$.

Palmowski, P., Rogowska-Wrzesinska, A., Williamson, J., Beck, H.C., Mikkelsen, J.D., Hansen, H.H., Jensen, O.N., 2014. Acute phencyclidine treatment induces extensive and distinct protein phosphorylation in rat frontal cortex. J. Proteome Res. 13, 1578-92. doi:10.1021/pr4010794

Plaisier, C.L., Pan, M., Baliga, N.S., 2012. A miRNA-regulatory network explains how dysregulated miRNAs perturb oncogenic processes across diverse cancers. Genome Res. 22, 2302-14. doi:10.1101/gr.133991.111

Poos, K., Smida, J., Nathrath, M., Maugg, D., Baumhoer, D., Korsching, E., 2013. How microRNA and transcription factor co-regulatory networks affect osteosarcoma cell proliferation. PLoS Comput. Biol. 9, e1003210. doi:10.1371/journal.pcbi.1003210

Powell, C.M., Miyakawa, T., 2006. Schizophrenia-relevant behavioral testing in rodent models: a 
uniquely human disorder? Biol. Psychiatry 59, 1198-207. doi:10.1016/j.biopsych.2006.05.008

Prabakaran, S., Swatton, J.E., Ryan, M.M., Huffaker, S.J., Huang, J.T.-J., Griffin, J.L., Wayland, M., Freeman, T., Dudbridge, F., Lilley, K.S., Karp, N.A., Hester, S., Tkachev, D., Mimmack, M.L., Yolken, R.H., Webster, M.J., Torrey, E.F., Bahn, S., 2004. Mitochondrial dysfunction in schizophrenia: evidence for compromised brain metabolism and oxidative stress. Mol. Psychiatry 9, 684-97, 643. doi:10.1038/sj.mp.4001511

$R$ Development Core Team, 2013. R: A language and environment for statistical computing. $R$ Foundation for Statistical Computing, Vienna, Austria. URL http://www.R-project.org/. R Found. Stat. Comput. Vienna, Austria.

Rajasekaran, A., Venkatasubramanian, G., Berk, M., Debnath, M., 2015. Mitochondrial dysfunction in schizophrenia: pathways, mechanisms and implications. Neurosci. Biobehav. Rev. 48, 10-21. doi:10.1016/j.neubiorev.2014.11.005

Ramnani, N., Owen, A.M., 2004. Anterior prefrontal cortex: insights into function from anatomy and neuroimaging. Nat. Rev. Neurosci. 5, 184-94. doi:10.1038/nrn1343

Rössler, W., Salize, H.J., van Os, J., Riecher-Rössler, A., 2005. Size of burden of schizophrenia and psychotic disorders. Eur. Neuropsychopharmacol. 15, 399-409. doi:10.1016/j.euroneuro.2005.04.009

Roweis, S.T., Saul, L.K., 2000. Nonlinear dimensionality reduction by locally linear embedding. Science 290, 2323-6. doi:10.1126/science.290.5500.2323

Sams-Dodd, F., 1996. Phencyclidine-induced stereotyped behaviour and social isolation in rats: a possible animal model of schizophrenia. Behav. Pharmacol. 7, 3-23.

Sarnyai, Z., Alsaif, M., Bahn, S., Ernst, A., Guest, P.C., Hradetzky, E., Kluge, W., Stelzhammer, V., Wesseling, H., 2011. Behavioral and molecular biomarkers in translational animal models for neuropsychiatric disorders. Int. Rev. Neurobiol. 101, 203-38. doi:10.1016/B978-0-12-3877185.00008-0

Schlicker, A., Domingues, F.S., Rahnenführer, J., Lengauer, T., 2006. A new measure for functional similarity of gene products based on Gene Ontology. BMC Bioinformatics 7, 302. doi:10.1186/1471-2105-7-302

Smith, J.W., Gastambide, F., Gilmour, G., Dix, S., Foss, J., Lloyd, K., Malik, N., Tricklebank, M., 2011. A comparison of the effects of ketamine and phencyclidine with other antagonists of the NMDA receptor in rodent assays of attention and working memory. Psychopharmacology (Berl). 217, 255-69. doi:10.1007/s00213-011-2277-5

Speer, N., Frohlich, H., Spieth, C., Zell, A., 2005. Functional grouping of genes using spectral clustering and Gene Ontology, in: Neural Networks, 2005. IJCNN '05. Proceedings. 2005 IEEE International Joint Conference on. pp. 298-303. doi:10.1109/IJCNN.2005.1555846

Stelzl, U., Worm, U., Lalowski, M., Haenig, C., Brembeck, F.H., Goehler, H., Stroedicke, M., Zenkner, M., Schoenherr, A., Koeppen, S., Timm, J., Mintzlaff, S., Abraham, C., Bock, N., Kietzmann, S., Goedde, A., Toksöz, E., Droege, A., Krobitsch, S., Korn, B., Birchmeier, W., Lehrach, H., Wanker, E.E., 2005. A human protein-protein interaction network: A resource for annotating the proteome. Cell 122, 957-968. doi:10.1016/j.cell.2005.08.029

Stewart, A.M., Kalueff, A. V, 2015. Developing better and more valid animal models of brain disorders. Behav. Brain Res. 276, 28-31. doi:10.1016/j.bbr.2013.12.024

Sullivan, P.F., Kendler, K.S., Neale, M.C., 2003. Schizophrenia as a complex trait: evidence from a meta-analysis of twin studies. Arch. Gen. Psychiatry 60, 1187-92. doi:10.1001/archpsyc.60.12.1187

Tandon, R., Keshavan, M.S., Nasrallah, H.A., 2008. Schizophrenia, "just the facts" what we know in 2008. 2. Epidemiology and etiology. Schizophr. Res. 102, 1-18. doi:10.1016/j.schres.2008.04.011

Tomasik, J., Rahmoune, H., Guest, P.C., Bahn, S., 2014. Neuroimmune biomarkers in schizophrenia. Schizophr. Res. doi:10.1016/j.schres.2014.07.025

Tomasik, J., Schultz, T.L., Kluge, W., Yolken, R.H., Bahn, S., Carruthers, V.B., 2015. Shared Immune 
and Repair Markers During Experimental Toxoplasma Chronic Brain Infection and Schizophrenia. Schizophr. Bull. doi:10.1093/schbul/sbv134

Tordjman, S., Drapier, D., Bonnot, O., Graignic, R., Fortes, S., Cohen, D., Millet, B., Laurent, C., Roubertoux, P.L., 2007. Animal models relevant to schizophrenia and autism: validity and limitations. Behav. Genet. 37, 61-78. doi:10.1007/s10519-006-9120-5

Torrey, E.F., Webster, M., Knable, M., Johnston, N., Yolken, R.H., 2000. The Stanley Foundation brain collection and Neuropathology Consortium. Schizophr. Res. 44, 151-155. doi:10.1016/S09209964(99)00192-9

Tsai, G., Coyle, J.T., 2002. Glutamatergic mechanisms in schizophrenia. Annu. Rev. Pharmacol. Toxicol. 42, 165-79. doi:10.1146/annurev.pharmtox.42.082701.160735

Turck, C.W., Maccarrone, G., Sayan-Ayata, E., Jacob, A.M., Ditzen, C., Kronsbein, H., Birg, I., Doertbudak, C.-C., Haegler, K., Lebar, M., Teplytska, L., Kolb, N., Uwaje, N., Zollinger, R., 2005. The quest for brain disorder biomarkers. J. Med. Invest. 52 Suppl, 231-5.

Wesseling, H., Gottschalk, M.G., Bahn, S., 2015a. Targeted multiplexed selected reaction monitoring analysis evaluates protein expression changes of molecular risk factors for major psychiatric disorders. Int. J. Neuropsychopharmacol. 18. doi:10.1093/ijnp/pyu015

Wesseling, H., Guest, P.C., Lee, C.-M., Wong, E.H., Rahmoune, H., Bahn, S., 2014. Integrative proteomic analysis of the NMDA NR1 knockdown mouse model reveals effects on central and peripheral pathways associated with schizophrenia and autism spectrum disorders. Mol. Autism 5, 38. doi:10.1186/2040-2392-5-38

Wesseling, H., Rahmoune, H., Tricklebank, M., Guest, P.C., Bahn, S., 2015b. A Targeted Multiplexed Proteomic Investigation Identifies Ketamine-Induced Changes in Immune Markers in Rat Serum and Expression Changes in Protein Kinases/Phosphatases in Rat Brain. J. Proteome Res. 14, 411-421. doi:10.1021/pr5009493

Wesseling, H., Want, E.J., Guest, P.C., Rahmoune, H., Holmes, E., Bahn, S., 2015c. Hippocampal Proteomic and Metabonomic Abnormalities in Neurotransmission, Oxidative Stress, and Apoptotic Pathways in a Chronic Phencyclidine Rat Model. J. Proteome Res. 14, 3174-87. doi:10.1021/acs.jproteome.5b00105

WHO | The global burden of disease: 2004 update, n.d.

Wong, A.H.C., Josselyn, S.A., 2015. Caution When Diagnosing Your Mouse with Schizophrenia: The Use and Misuse of Model Animals for Understanding Psychiatric Disorders. Biol. Psychiatry 79, 32-38. doi:10.1016/j.biopsych.2015.04.023

Zanzoni, A., Montecchi-Palazzi, L., Quondam, M., Ausiello, G., Helmer-Citterich, M., Cesareni, G., 2002. MINT: a Molecular INTeraction database. FEBS Lett. 513, 135-140. doi:10.1016/S00145793(01)03293-8

Zare, H., Kaveh, M., Khodursky, A., 2011. Inferring a Transcriptional Regulatory Network from Gene Expression Data Using Nonlinear Manifold Embedding. PLoS One 6, e21969. doi:10.1371/journal.pone.0021969 